\title{
Ceftriaxone-induced hemolytic anemia in a child successfully managed with intravenous immunoglobulin
}

\author{
Aysel Vehapoğlu ${ }^{1}$, Nilüfer Göknar ${ }^{1}$, Rümeysa Tuna ${ }^{1}$, Fatma Betül Çakır² \\ ${ }^{1}$ Department of Pediatrics, ${ }^{2}$ Division of Pediatric Hematology-Oncology, Bezmialem Vakıf University Faculty of Medicine, \\ Istanbul, Turkey. E-mail: ayvahap@hotmail.com \\ Received: 11 May 2015, Revised: 25 August 2015, Accepted: 7 September 2015
}

\begin{abstract}
SUMMARY: Vehapoğlu A, Göknar N, Tuna R, Çakır FB. Ceftriaxoneinduced hemolytic anemia in a child successfully managed with intravenous immunoglobulin. Turk J Pediatr 2016; 58: 216-219.

Drug-induced hemolytic anemia is an immune-mediated phenomenon that leads to the destruction of red blood cells. Here, we present a case of lifethreatening ceftriaxone-induced hemolytic anemia (CIHA) in a previously healthy 3-year-old girl. We also reviewed the literature to summarize the clinical features and treatment of hemolytic anemia. Acute hemolysis is a rare side effect of ceftriaxone therapy associated with high mortality. Our patient had a sudden loss of consciousness with macroscopic hematuria and her hemoglobin dropped from 10.2 to $2.2 \mathrm{~g} / \mathrm{dl}$ over 4 hours, indicating that the patient had life-threatening hemolysis after an intravascular dose of ceftriaxone who had previously been treated with ceftriaxone in intramuscular form for six days. CIHA is associated with a positive direct antiglobulin test, revealing the presence of IgG in all cases and C3d in most cases. Our patient's direct antiglobulin test was positive for IgG $(3+)$ and for C3d $(4+)$. The case was managed successfully with supportive measures and intravenous immunoglobulin therapy. Ceftriaxone is used very frequently in children; an early diagnosis and proper treatment of hemolytic anemia are essential to improve the patient outcome. The pathophysiological mechanism is the same as for non-drug autoimmune hemolytic anemia. However, there is still no consensus treatment for CIHA. Intravenous immunoglobulin can be used in clinical emergencies, such as our case, or in refractory cases.
\end{abstract}

Key words: ceftriaxone, immune hemolytic anemia, IVIG, children.

Drug-induced immune hemolytic anemia (DIIHA) is rare and a specialized laboratory is often needed to perform the serological tests to confirm diagnosis. Although there are no good incidence data, rough estimates suggest that the incidence is around one per million population ${ }^{1}$. A PubMed literature search using the keywords "drug-induced immune hemolytic anemia" identified 430 articles on the subject published from 1966 to January 2015. Ceftriaxoneinduced hemolytic anemia has been reported 37 times (including our case) between 1991 and 2015 (PubMed online search: ceftriaxone and hemolysis, ceftriaxone-induced hemolytic anemia). Of these 37 patients, 27 (73\%) were younger than 18 years. To date, about 130 drugs have been implicated in causing a positive direct antiglobulin test (DAT or Coombs test) and hemolytic anemia, with three groups of drugs predominating: antimicrobials (42\%); anti-inflammatory drugs (15\%); and antineoplastics (11\%). The drugs associated most frequently with hemolytic anemia were piperacillin, cefotetan, and ceftriaxone. Twelve cephalosporins have been reported to cause DIIHA; primarily cefotetan and ceftriaxone have been associated with mortality ${ }^{2}$. There is no consensus on the management of DIIHA. We report a life-threatening reaction to ceftriaxone in a previously healthy child who was treated successfully with intravenous immunoglobulin (IVIG).

\section{Case Report}

A 3-year-old female, who had no relevant history of drug allergies, was transferred to our hospital for further treatment of a sudden episode of 
macroscopic hematuria, hemoglobinuria, and hemolytic anemia that developed after being given seventh dose of ceftriaxone. The previous week, she had complained of a cough and fever and had been diagnosed with pneumonia. She had been treated with ceftriaxone ( $1 \mathrm{~g} /$ day intramuscularly) for 6 days as an outpatient at another center. Her symptoms worsened and the fever persisted for 6 days. Her blood tests were checked on the seventh day. The complete blood count included hemoglobin $10.2 \mathrm{~g} / \mathrm{dl}$, hematocrit $29 \%$, white blood cell count 9,300/ $\mu \mathrm{l}$, and platelet count $338,000 / \mu \mathrm{l}$. She was hospitalized and continued on IV ceftriaxone. She had sudden-onset pallor, fatigue, and hemoglobinuria within about 30 minutes of starting the seventh dose of IV ceftriaxone. The patient had not received any medications other than ceftriaxone. On admission to our hospital, she was unconscious and was transferred to our pediatric intensive care unit. Her vital signs were heart rate $160 / \mathrm{min}$, respiratory rate $40 / \mathrm{min}$, temperature $36.8^{\circ} \mathrm{C}$, and blood pressure $80 / 60 \mathrm{~mm} \mathrm{Hg}$. She had very pale conjunctivas and mucous membranes. The spleen was palpable $2 \mathrm{~cm}$ below the costal margin. She was given IV fluids and the initial laboratory tests revealed severe anemia with hemoglobin $(\mathrm{Hb}) 2.2 \mathrm{~g} / \mathrm{dl}$ and hematocrit $4.3 \%$. The reticulocyte count was $2.74 \%$. The peripheral blood smear was consistent with 4 (+) hemolysis, having numerous schistocytes and nucleated red blood cells. The blood chemistry revealed indirect bilirubinemia (1.12 $\mathrm{mg} / \mathrm{dl}$; normal range: 0-0.6) and elevated lactate dehydrogenase (1125 U/1; normal range: 125220). The haptoglobin level was $<7.56$ (normal 30-200) and Coombs' direct antiglobulin test was positive for $\operatorname{IgG}(3+)$ and for C3d $(4+)$. The activated partial thromboplastin and prothrombin times were normal. The liver and renal function tests and serum electrolytes were normal. Drug-dependent antibody testing was not available in our hospital. The reddish urine was $4(+)$ for hemoglobin. DIIHA was diagnosed and the ceftriaxone stopped. The patient was treated with one unit of packed red blood cells, high-dose immunoglobulin $(0.4 \mathrm{~g} / \mathrm{kg})$, and supportive care, including oxygen and nutritional support. She improved quickly and her $\mathrm{Hb}$ increased from 2.2 to $7.3 \mathrm{~g} / \mathrm{dl}$. Although she had prolonged gross hemoglobinuria, her $\mathrm{Hb}$ did not drop again nor did she develop acute tubular necrosis. She was discharged on the fourth hospital day with $\mathrm{Hb} 12.1 \mathrm{~g} / \mathrm{dl}$ and normal blood chemistry. The patient had no further hemolytic episodes. The glucose-6-phospate dehydrogenase level and osmotic fragility testing were normal. Her hematocrit remained stable over the following 9 months. She was warned never again to take cephalosporin group antibiotics.

\section{Discussion}

Ceftriaxone, a third-generation cephalosporin, is a broad spectrum antibiotic commonly used to treat Gram-positive and -negative bacteria since 1982. However, physicians should be aware of the many potential adverse effects of ceftriaxone, including elevated liver enzyme levels in $3 \%$ of patients, diarrhea in $2.7 \%$, leukopenia in $2.1 \%$, and hypersensitivity reactions in $1.7 \%$. Other rare $(<0.1 \%)$, but serious, complications include: agranulocytosis, allergic pneumonitis, anaphylaxis, biliary lithiasis, colitis, including Clostridium difficile colitis, seizures, serum sickness, and StevensJohnson syndrome $e^{3}$. Second and third generation cephalosporins are the leading causes of druginduced hemolytic anemia. Of these cases, $20 \%$ have been attributed to ceftriaxone, especially in children who previously received the drug, as in our case. Reported cases of cardiovascular decompensation and renal failure in pediatric CIHA patients have a high mortality rate. Garratty et $\mathrm{al}^{4}$ reported the first case of immune hemolytic anemia induced by ceftriaxone in 1991; their patient died of hemolysis and acute renal failure.

Drug-induced immune hemolytic anemia occurs primarily as a result of drug-induced antibodies, either drug-dependent (penicillin or immune complex type) or drug-independent (autoantibodies). The suggested mechanism for DIIHA is the production of drug-independent antibodies (DIABs), which can be detected in vitro without the addition of any drug, similar to red blood cell (RBC) autoantibodies. Fludarabine, methyldopa, $\beta$-lactamase inhibitors, and platinum-based chemotherapeutics are the drugs that most commonly induce autoantibody formation. The main antibody produced is IgG. ${ }^{5}$ The second suggested mechanism for DIIHA is drug-dependent antibody (DDABs) formation that reacts in vitro only in the presence of the 
drug. These are likely combinations of drug or drug metabolite plus RBC membrane proteins. An example of this would be hemolysis caused by penicillin or cefotetan, ceftriaxone and piperacillin (immune complex type). The antibodies formed can be either the IgG or IgM subtype. The immune complexes bind nonspecifically to the RBC membranes and activate complement, which destroys RBCs. Drug-dependent antibodies have been detected in healthy donors. Detectable antibodies to ceftriaxone are necessary, but not sufficient, to cause hemolysis in CIHA. Studies found anti-ceftriaxone antibodies in 8 of 64 pediatric patients with human immunodeficiency virus infection and sickle cell disease exposed to ceftriaxone ${ }^{6}$. Only two of these eight patients with antibodies experienced hemolysis. Drugdependent antibodies usually cause a positive DAT and negative elution test; while drugindependent antibodies are indistinguishable serologically from warm autoantibodies and are similarly steroid-responsive ${ }^{6}$. Some patients have combinations of these antibodies. It has been reported that $65 \%$ of the patients with ceftriaxone-induced immune hemolytic anemia have often received the drug previously. Hemolytic anemia is more acute and severe in children compared to adults. ${ }^{7}$ Reactions in children can start 5 minutes to 7 days after receiving the drug. The reaction in our patient started 30 minutes after ceftriaxone administration. The clinical presentation of CIHA is usually abrupt, with sudden-onset pallor, tachypnea, dyspnea, vomiting, headache, and lumbar or abdominal pain. In serious cases, severe anemia results in hypovolemic shock and cardiorespiratory arrest. Massive intravascular hemolysis and hemoglobinuria can progress to acute tubular necrosis. The mortality rate was $30 \%$ in all age groups and $64 \%$ in children. Cardiovascular decompensation and renal failure in pediatric patients are associated with high mortality rates. The majority of patients had underlying conditions $(70 \%)$, of which sickle cell disease and glucose-6phosphate dehydrogenase deficiency were most common. Common features included elevated lactate dehydrogenase $(70 \%)$; early, new-onset hemoglobinuria (59\%); acute renal failure (46\%); a positive DAT $(70 \%)$; and anti-ceftriaxone antibodies $(68 \%)^{8}$. Our patient had clinical evidence of intravascular hemolysis characterized by a striking decline in the hemoglobin concentration and severe hemoglobinuria, but she did not develop renal tubular necrosis. The resulting hemoglobinuria could be nephrotoxic, particularly when intratubular obstruction facilitates proximal tubular heme uptake. Our case met the diagnostic criteria for autoimmune hemolytic anemia (AIHA); e.g., the clinical and serological criteria for hemolysis (decreasing hemoglobin level, elevated lactate dehydrogenase level, low haptoglobin level, and a peripheral blood smear consistent with hemolysis) along with a positive Coombs test and IgG autoantibodies (warm antibodies). Arndt et al. ${ }^{9}$ reported a series of 25 DIIHA cases, all of whom were reactive with anti-C3 and $47 \%$ were reactive with anti-IgG due to ceftriaxone.

Management generally consists of discontinuing the ceftriaxone, while providing cardiopulmonary support and administering RBC transfusions. The treatment of patients with AIHA remains a challenge. Blood can usually be transfused safely because the antibodies are drug dependent. There is no compelling evidence that steroids are effective in treating hemolytic anemia with drug-dependent antibodies. Steroid therapy is of questionable efficacy in the treatment of CIHA $^{10}$. Zanetti et al. ${ }^{11}$ reported that a patient with DIIHA received a total of eight units of packed RBCs and intravenous methylprednisolone, but continued to show signs of hemolysis; subsequently, she was given intravenous immunoglobulin (IVIG) and did not require any blood products during follow-up. In a mouse model, Chen et al. ${ }^{12}$ demonstrated that AIHA-causing antibodies are an important factor to consider in the response to IVIG therapy. The pathophysiological mechanism is the same for non-drug AIHA. Intravenous immunoglobulin can be used in clinically emergent cases, such as our case, or refractory cases. Studies reported that hematologic recovery commonly occurs within 2 weeks, but the DAT can remain positive for weeks or months. In our case, hematological recovery occurred the second day and the child was discharged home 4 days after the IVIG infusion. Although she had prolonged gross hemoglobinuria, her $\mathrm{Hb}$ did not drop again, she nor did she develop acute tubular necrosis. 


\section{Acknowledgement}

The authors of this manuscript would like to thank the mother and child.

\section{REFERENCES}

1. Chen F, Zhan Z. Severe drug-induced immune haemolytic anaemia due to ceftazidime. Blood Transfus 2014; 12: 435-437.

2. Garratty G, Arndt PA. Drugs that have been shown to cause drug-induced immune hemolytic anemia or positive direct antiglobulin tests: some interesting findings since 2007. Immunohematology 2014; 30: 66-79.

3. Rocephin (ceftriaxone sodium) injection, powder, for solution [Genentech, Inc].

4. Arndt PA. Drug-induced immune hemolytic anemia: the last 30 years of changes. Immunohematology 2014; 30: $44-54$.

5. Leger RM, Arndt PA, Garratty G. How we investigate drug-induced immune hemolytic anemia. Immunohematology 2014; 30: 85-94.

6. Quillen K, Lane C, Hu E, Pelton S, Bateman S. Prevalence of ceftriaxone-induced red blood cell antibodies in pediatric patients with sickle cell disease and human immunodeficiency virus infection. Pediatr Infect Dis J 2008; 27: 357-358.
7. Pierce A, Nester T. Education Committee of the Academy of Clinical Laboratory Physicians and Scientists. Pathology consultation on drug-induced hemolytic anemia. Am J Clin Pathol 2011; 136: 7-12.

8. Northrop MS, Agarwal HS. Ceftriaxone-induced hemolytic anemia: case report and review of literature. J Pediatr Hematol Oncol 2015; 37: e63-e66.

9. Arndt PA, Leger RM, Garratty G. Serologic characteristics of ceftriaxone antibodies in 25 patients with druginduced immune hemolytic anemia. Transfusion 2012; 52: $602-612$.

10. Liu W, Yu D. Adverse drug reactions during ceftriaxone treatment can cause severe hemolysis. Pediatr Allergy Immunol 2014; 25: 101-102.

11. Zanetti RC, Biswas AK. Hemolytic anemia as a result of piperacillin/tazobactam administration: a case report and discussion of pathophysiology. Mil Med 2013; 178: 10451047.

12. Chen X, Ghaffar H, Jen CC, et al. Antibody specific for the glycophorin A complex mediates intravenous immune globulin-resistant anemia in a murine model. Transfusion 2014; 54: 655-664 